\title{
IMPACTO DEL AISLAMIENTO SOCIAL EN MIPYMES DEL CALZADO EN COLOMBIA OCASIONADO POR CHOQUE EXÓGENO (SARS COV-2)
}

\section{IMPACT OF SOCIAL ISOLATION ON MSMES OF FOOTWEAR IN COLOMBIA CAUSED BY EXOGENOUS SHOCK (SARS COV-2)}

Recibido: 23 de septiembre de 2020

Evaluado: 25 de octubre de 2020

Aprobado: 11 de diciembre de 2020

\author{
Iván Darío Medina Rojas* \\ Universidad de la Salle \\ Orcid: https://orcid.org/0000-0002-8521-5005 \\ Eliana Patricia Sánchez Parra ${ }^{\star *}$ \\ Universidad De Celaya, México \\ Orcid: https://orcid.org/0000-0003-1861-9111
}

Yedy Alejandra Solís Montaño ${ }^{\star \star \star}$

Universidad Minuto de Dios

Orcid: https://orcid.org/0000-0002-3691-6817

Cómo citar este artículo: Medina Rojas, I.D., Sánchez Parra, E.P., y Solís Montaño, Y.A. (2021). IMPACTO DEL AISLAMIENTO SOCIAL EN MIPYMES DEL CALZADO EN COLOMBIA OCASIONADO POR CHOQUE EXÓGENO (SARS COV-2). Revista Estrategia Organizacional, 10(1), https://doi.org/10.22490/25392786.456

* $\quad$ ADocente Corporación Universitaria Minuto de Dios, UNIMINUTO. Estudiante doctorado Agrociencias, MSc. Desarrollo Sostenible y Medio Ambiente. Profesional: Economista. Microbiólogo Industrial. Correo electrónico: imedina@uniminuto.edu

** Docente Corporación Universitaria Minuto de Dios, UNIMINUTO. Candidata doctorado Administración, MSc. Innovación para el Desarrollo Empresarial.Profesional:Administradora de Empresas.Correo electrónico: epsanchez@ uniminuto.edu

*** Docente Corporación Universitaria Minuto de Dios, UNIMINUTO. MSc. Ciencia Política. Profesional en Relaciones Económicas Internacionales. Correo electrónico: ysolis@uniminuto.edu 


\title{
RESUMEN
}

Introducción: se propende por realizar una aproximación al choque exógeno ocasionado por el SARS CoV-2 y su afectación en las MiPymes del calzado en Colombia, tomando una radiografía una vez se determinan medidas de distanciamiento social. Las empresas se ven afectadas negativamente por la cuarentena y el impacto generado por la incertidumbre toma parte en las decisiones, reduciendo la capacidad de predecir resultados con precisión. Metodología: de manera descriptiva, utilizando un cuestionario semiestructurado y mediante vía telefónica, se establece contacto con 34 MiPymes del calzado (ubicadas en las ciudades de Bogotá, Cali y Cúcuta); se indagan aspectos relacionados con la operación y gestión en medidas de confinamiento, de igual manera, sobre propuestas de avance. Resultados: más de tres cuartas partes de las empresas no han podido operar de la misma forma una vez se fijó el decreto de cuarentena, el rendimiento en producción y ventas sufrió un marcado decrecimiento, mermando el flujo de efectivo y reduciendo la capacidad de maniobra de las empresas. Las MiPymes del sector tienen un escenario muy complejo por lo que es importante determinar estrategias que permitan llevar la situación. Resultados: dado que el impacto en el sector ha sido marcado por pérdidas considerables, las estrategias basadas en modelos colaborativos, de trabajo en red, con articulación entre partes interesadas (sector público, privado y agremiaciones) resultan necesarias para reducir el grado de afectación; sumado a ello, el apoyo en las nuevas tecnologías como el marketing digital, o en la creación de una tienda multimarca, posiblemente permitirá revertir el fenómeno adverso.

Palabras clave: MiPymes; SARS CoV-2; Incertidumbre económica; Calzado; Asociatividad empresarial.

\begin{abstract}
Introduction: It seeks an approach to the exogenous shock caused by SARS CoV-2 and the degree to which it affected the MSMES (Micro, Small and Medium Enterprises) of the footwear industry in Colombia, by taking a radiography once social distancing rules are determined. Companies are negatively affected by quarantine and the impact generated by uncertainty takes part in decisions, reducing the ability to accurately predict results with precision. Methodology: In a descriptive way, and using a semi-structured questionnaire via phone, contact is made with 34 footwear MSMEs (located in the cities of Bogotá, Cali and Cúcuta); different aspects related to the operation and process of lockdown measures are explored as well as an implementation plan proposal. Results: Over three quarters of the enterprises haven't been able to operate as
\end{abstract}


they did before quarantine rules were established. Production and sales performance have suffered a significant decrease, which has reduced the cash flow and the ability to adapt their supply chain and production processes. MSMES of the sector have a very complex scenario so it's important to determine strategies that allow them to manage the situation. Conclusions: Since the footwear industry has been badly affected by considerable losses, strategies based on collaborative models and networking with an interface among the parts (private sector, public sector and labor unions) are necessary to reduce the high impact. Moreover, strategies such us supporting new technologies like the digital marketing and creating a multi brand store might help to restore this adverse effect.

Keywords: MSMEs; SARS CoV-2; Economic uncertainty; Footwear; Business associativity.

\section{INTRODUCCIÓN}

La alta replicación del SARS CoV-2 y su afectación en la salud del ser humano derivó en una crisis sanitaria global con impactos serios en la economía y gran angustia social, la cual requiere de acciones efectivas por parte de los gobiernos, las personas y las empresas (OMS, 2020). Se evidencia un incremento en la incertidumbre en temas inherentes a la salud, la gestión pública, la industria, el medio ambiente, la energía y el impacto a futuro que pueda traer consigo la presencia del agente vírico (Shah, et al., 2020) (Elavarasan \& Pugazhgendhi, 2020); no obstante, y de acuerdo con (Hartmann \& Lussier, 2020), para entender los fenómenos ocurridos bajo la actual coyuntura, han de ser observados y analizados mediante perspectivas multidisciplinares e interdisciplinares; comprendiendo por ejemplo, las múltiples aristas de afectación en el desenvolvimiento económico, ya que en las relaciones comerciales, es implícita la comunicación y la interacción social, en la que patrones de movilidad se encuentran presentes. En el curso de la aparición del nuevo coronavirus, el nivel de producción ha bajado considerablemente, países desarrollados enfrentan alta inflación, marcado desempleo y reducción en el crecimiento del Producto Interno Bruto (Chakraborty \& Maity, 2020); el impacto en países en vías de desarrollo es aún más fuerte, y si se desea establecer un parangón, la afectación económica y social es similar a la crisis griega de fines de la primera década del nuevo siglo (Kottika, et al., 2020). 
A su vez, el bastión de la economía mundial ha recaído en las micro, pequeñas y medianas empresas "MiPymes", por lo que el evento pandémico actual resquebraja sus cimientos, alterando una base débil por esencia, dado su tamaño y limitación de recursos, incrementando su vulnerabilidad (Eggers, 2020). A priori de lo observado en el presente, las MiPymes eran referidas como las victimas por excelencia de una crisis económica prolongada, factores como recursos financieros limitados, dependencia de préstamo a bancos con altas tasas de interés, deficiencia en capacidades tecnológicas, gerenciales y humanas; sin embargo, una inversión en ventas y marketing puede mejorar su desempeño en periodos de recesión económica (Bourletidis \& Triantafyllopoulos, 2014); a posteriori al choque exógeno, asumido como una perturbación en la capacidad de maniobra de la MiPyme trae un incremento en la incertidumbre, la cual correlaciona una afectación de las finanzas empresariales fruto del distanciamiento social, propiciando también un distanciamiento financiero (Brown \& Rocha, 2020).

A pesar del conjunto de inconvenientes que enfrentan las MiPymes, ciertos elementos podrían minimizar el impacto de las crisis; por su tamaño, este tipo de empresas presentan mayor flexibilidad cuando se asoman oportunidades o amenazas en el entorno, acá la toma de decisiones suele ser más rápida, siendo una herramienta clave ante episodios de desasosiego. También, se han utilizado contingencias para minimizar el impacto negativo, tales como las tecnologías digitales (tecnologías móviles, internet de las cosas, bigdata, inteligencia artificial y blockchain), las cuales mejoran la competitividad, productividad y actuación; más aún cuando el evento es disruptivo, y termina afectando el negocio y la cadena de suministro, mermando la eficiencia, la rentabilidad y poniendo en riesgo la supervivencia de la MiPyme, por lo que cierta infraestructura informática es necesaria (Papadopoulos, Baltas, \& Balta, 2020). Sumado a ello, es importante revisar el tipo de empresa, por ejemplo, si es familiar o no, ya que como afirma (Eggers, 2020), las empresas familiares suelen fijar metas a largo plazo, queriendo mantener su funcionamiento por más de una generación, aunque presenten mayor aversión al riesgo.

El presente escrito toma como referencia el segmento MiPyme del calzado, un sector de talla mundial, que busca posicionarse con productos de alta calidad pero que a su vez ha tenido que enfrentar procesos de reingeniería para mitigar algunos impactos negativos, como el generado al medio ambiente, el incremento del comercio de productos chinos y la sobreproducción de 
cuero en América Latina, en países como Brasil (Cámara de Comercio de Bogotá, 2016) (Cámara de Comercio de Bogotá, 2019). De acuerdo con el reporte ¿Cómo va el sector? de la Asociación Colombiana de Industriales del Calzado el Cuero y sus Manufacturas "ACICAM" que realiza la evaluación del año 2019, concluye que, en cuanto al calzado, hubo una caída en la demanda interna, una disminución de los pedidos en las grandes superficies y tiendas especializadas, una reducción de las exportaciones y a una merma en la proyección de ventas deseadas (ACICAM, 2020). Es importante hacer hincapié en que los resultados son previos al evento pandémico, por lo que el reto se incrementa.

Precisamente, algunos de los elementos de la baja productividad del segmento en las MiPymes del calzado en la capital colombiana no son ajenos a los de otros sectores. Por ejemplo, una ausencia de información reduce capacidad de gestión y disminuye el poder de negociación, generando sobrecostos en la compra de materia prima; también, trae dificultades a la hora de comercializar la producción; el acceso a la tecnología y al crédito es restringido a causa de los ingresos bajos; y sumado a lo anterior, otros aspectos como la escasa destinación de recursos para capacitación, el problema del contrabando y la reiterada complicación por importación de calzado chino (Páez, Jiménez, \& Danna-Buitrago, 2018). De hecho, la tendencia en los últimos años es que la producción venga especialmente de la zona del sudeste asiático donde la fabricación se da a bajo costo, por lo que desde occidente se ha optado por ofrecer productos diferenciados, o por lo menos, donde la industria se encuentra madura como la italiana, escenario distinto al colombiano (Confente, Buratti, \& Russo, 2015).

En consecuencia, con lo anterior y viendo la importancia de esta industria en Colombia, se adelanta una investigación conjunta entre la Corporación Universitaria Minuto de Dios y algunos empresarios del sector del calzado, el cuero y la marroquinería, en la que, mediante ejercicios asociativos entre la academia y la industria se busca dinamizar este segmento de la economía, tales como ferias, charlas o capacitaciones. No obstante, la actual coyuntura trae un nuevo reto para la MiPyme de este fragmento de la economía nacional, por lo que se pretende develar la situación del momento en aras de buscar soluciones que reduzcan el impacto negativo por la limitación de operación observada en esas empresas. 


\section{METODOLOGÍA}

La investigación es de carácter descriptivo (Bairagi \& Munot, 2019), se pretende descubrir ciertas características o comportamientos al enfrentarse a un patrón particular (episodio pandémico derivado del nuevo coronavirus) sobre un grupo específico (empresarios de la industria del calzado); con los hallazgos encontrados se pretende comprender la problemática de la actual coyuntura. La información de fuentes primarias se recolecta mediante un cuestionario semiestructurado, empleando la herramienta formularios de Google; la encuesta fue realizada en el periodo comprendido entre los meses de mayo y junio de 2020. Se recolectó información vía telefónica de 34 MiPymes (14 empresas en Bogotá, 14 empresas en Cali y 6 empresas en Cúcuta), de un total de 80 seleccionadas para aplicar el instrumento, las 46 en las que no se pudo realizar, obedeció a criterios de imposibilidad al establecer la conexión, de no estar de acuerdo en suministrar información, o el establecimiento se encontraba cerrado.

\section{RESULTADOS Y DISCUSIÓN}

Es contundente y avasallador el avance del nuevo coronavirus a nivel mundial, las economías se han visto abocadas en realizar procesos de reingeniería; el comportamiento de replicación ha sido contundente y ha lacerado los cimientos de las empresas. La fracción perteneciente al sector del calzado no es la excepción; a pesar de ser uno de los 20 sectores eje del Programa de Transformación Productiva del Ministerio de Comercio, Industria y Turismo, fruto del crecimiento que ha tenido en los últimos dos lustros, aumento del empleo en este rubro y vocación exportadora, gracias a mejoras en innovación y calidad (Rivera, 2020).

La operación de la empresa durante las medidas de confinamiento

La evidencia muestra la sombría realidad que se constata a nivel mundial, del conjunto de empresas encuestadas, un contundente $77,8 \%$ no han podido operar de la misma forma una vez se instaura la cuarentena en el territorio nacional fijado a partir del 24 de marzo de 2020 en la medianoche, y a pesar de que paulatinamente ciertos sectores de la economía se han ido reactivando, el proceso ha sido lento por las medidas de aislamiento preventivo y distanciamiento social presentadas en los últimos meses; tan solo un 7,4\% de las empresas expresaron abiertamente que han podido sopesar la situación y mantienen producción y el 14,8\% restante no responde. 
Se establece un comparativo porcentual del rendimiento de las empresas antes y después del confinamiento. De ellas, el $69,6 \%$ para producción y el 78,3\% para ventas, se ubican en el rango más bajo de eficiencia ( $0 \%$ a $10 \%)$; si se amplía el rango, el escenario continua siendo muy similar; el $86,9 \%$ de las MiPymes mantiene un nivel de producción que no supera el $40 \%$, en tanto que el mismo porcentaje, no supera el $30 \%$ de ventas que se registraban antes de la pandemia y tan solo el 13,1\% se encuentra por encima de ese índice (ver gráfica 1A y 1B). Lo anterior indica que la gran mayoría de empresas presentaron una reducción significativa en su producción y ventas, lo cual hace necesario buscar medidas adicionales a las estipuladas por el Gobierno Nacional que les facilite salir de la crisis y evitar posibles liquidaciones o cierres temporales.

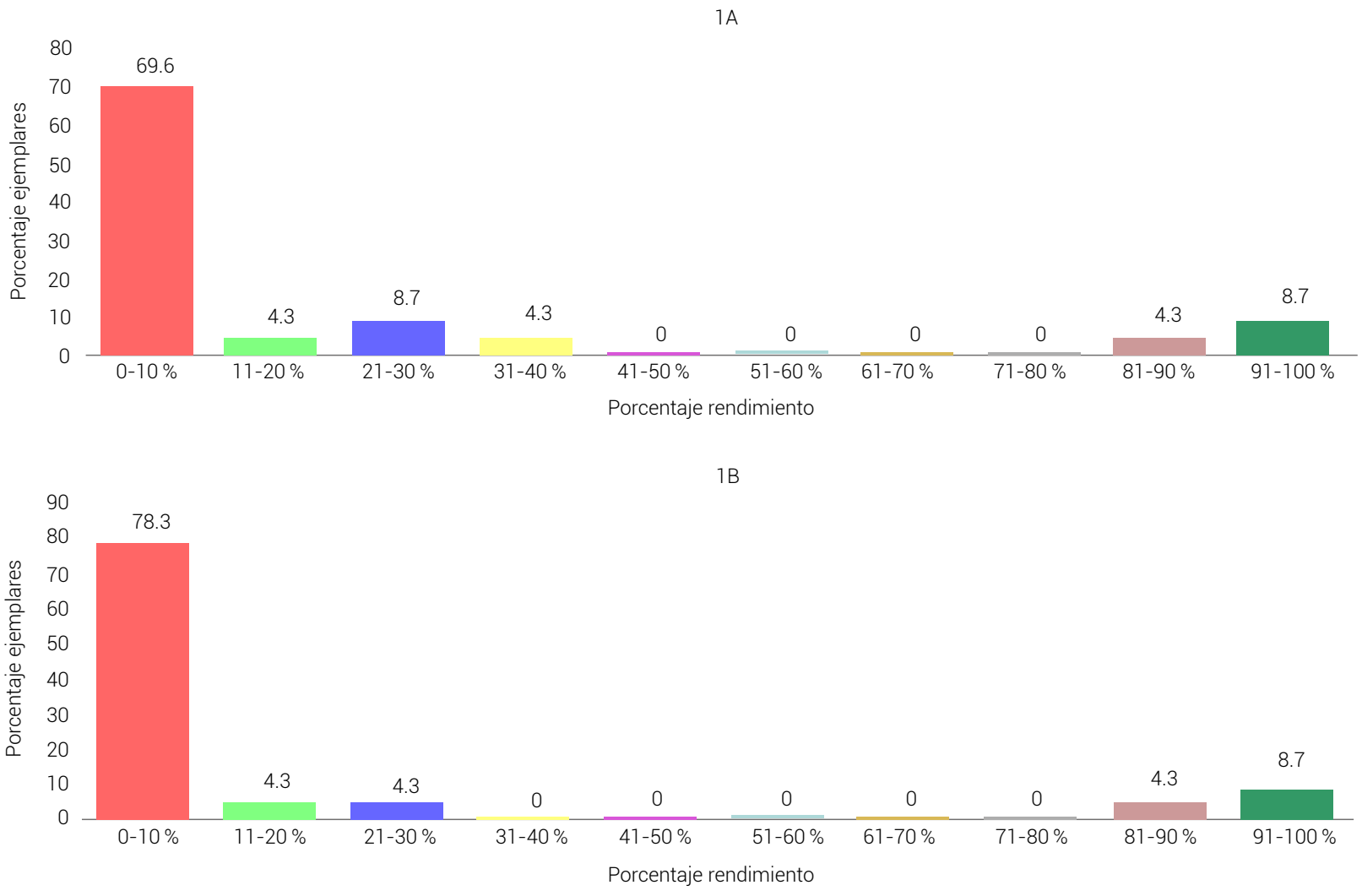

Figura 1. 1A) Rendimiento producción en escenario de aislamiento social. 1B) Rendimiento ventas en escenario de aislamiento social.

Fuente. Elaboración propia 
En las empresas consultadas se determinó que el 60,6\% han suspendido contratos o han solicitado a sus trabajadores que no asistan durante el periodo de cuarentena; el 39,4\% llegaron a acuerdos con sus trabajadores sin perder de vista la constante incertidumbre sobre el futuro del personal. Sumado a ello otro tema que reviste importancia es el de los arrendamientos, de los encuestados, el $19,2 \%$ no tienen problema con ello al realizar operaciones de producción en vivienda propia; el $23,1 \%$ paga por el momento solamente servicios públicos, pero la deuda continua; el 7,7\% paga fracción del arriendo y los servicios; otro 7,7\% no paga nada de momento, pero la deuda queda, lo que reviste gran preocupación; el 11,5\% no ha conseguido llegar a un punto de encuentro con el arrendador y el restante $30,8 \%$ no brinda esa información.

En cuanto a las opciones para mejorar la liquidez del negocio, la mayoría han recurrido a préstamos bancarios con tasa de interés preferencial $(52,2 \%)$, han recibido un subsidio por parte del gobierno para el pago de nómina (8,7\%), o familiares y amigos les han proporcionado un préstamo (8,7\%), como elementos reiterativos, el 30,4\% restante no suministra información sobre este tema. De esta manera se hace palpable la dificultad que tiene las MiPymes para asumir sus costos, tales como la nómina, ocasionado por la pérdida en el flujo de efectivo; muchas de estas empresas dependen entonces de los mercados de crédito, y dada su vulnerabilidad, los bancos pueden solicitar garantías adicionales, el dilema es que al no tener suficientes activos, las garantías escasean y pueden ser objeto de restricciones financieras (Mol-Gómez-Vázquez, Hernández-Cánovas, \& Köeter-Kant, 2020). De acuerdo con cifras emitidas por la Asociación Colombiana de las Micro, Pequeñas y Medianas Empresas "ACOPI", en el informe "Tablero de control impacto del Covid-19 en las MiPymes de Bogotá" con fecha corte 21 de junio 2020, el sector del calzado se situó en uno de los más afectados por su reducción en la mano de obra, con un porcentaje del 64,9\% (ACOPI, 2020).

\section{Gestión en épocas de pandemia}

Dentro de las categorías de actividades primarias que se localizan en la esfera de las MiPymes del calzado, se pretendió indagar sobre soluciones transitorias en materia de ingresos de la empresa y personales, sobre necesidades en el hogar, y mecanismos para mantener el empleo y las operaciones de la empresa. A nivel personal, los empresarios han recurrido a recursos propios para llevar la situación, pero estos se han encogido en el tiempo, por lo que se recurre a préstamos 
familiares o bancarios; las familias de los empleados han tenido que disminuir consumos, reestructurar el gasto del hogar y en algunos casos, enviar a su familia a otros municipios del país. Frente al manejo en la empresa, existe un apalancamiento mediante préstamos bancarios, pero también han dado una mirada hacia canales virtuales (vender online, abrir página web, vender en redes como WhatsApp, Facebook o Instagram) al fabricar productos acordes a las necesidades de los clientes, o mediante ventas a domicilio de contado.

Asimismo, las empresas necesitan mantener el empleo y sus operaciones. Nuevamente recurrir a préstamos bancarios es una de las opciones más utilizadas, no obstante, algunos han logrado la operacionalización con ahorros propios, aunque para muchos empresarios no es sostenible, la capacidad de maniobra se reduce a 15-20 días, de ahí en adelante suele ser más complicado, hasta el punto de enviar a vacaciones a los trabajadores, cesar contratos o en el peor de los casos, cerrar algunas fábricas. En adición, el panorama que tienen los empresarios de sus homólogos es que el sector se ha visto seriamente golpeado, de establecimientos que conocen o han tenido algún tipo de relacionamiento, comentan que es muy común ver cierres temporales o cierres definitivos; quienes continúan operando demuestran ingresos reducidos, otros se han podido mantener gracias a la experiencia cosechada o porque tienen un volumen considerable de clientes. Es importante hacer hincapié que, ajena al tipo de crisis, las MiPymes enfrentan retos por sus restricciones de acceso a capital, y ausencia de experiencia en gestión y talento (Kottika, et al., 2020).

\section{Propuesta de avance}

En cuanto a una gestión individual o asociativa, el 65\% de los encuestados prefieren mantenerse independientes de cualquier proceso colectivo, en tanto que el 35\% están interesados en realizar algún tipo de sociedad. Respuestas como "nunca me han gustado las asociaciones", "no lo he contemplado", "el proceso asociativo es muy complicado", "nada personal, solo prefiero trabajar individualmente" o "porque no sirve trabajar en sociedad" demuestra cierta reticencia a generar cooperativas, asociaciones de empresarios, consorcios u otro tipo de configuración de trabajo conjunto; por otra parte, contestaciones como "la unión hace la fuerza, individualmente se llega más rápido, pero acompañado se llega más lejos", "He recibido respaldo de la Cooperativa", o "se cosechan frutos por el accionar de algunas asociaciones", mantienen posturas positivas de establecer alianzas. 
En conexión con lo anterior, la literatura habla de modelos de colaboración como son las asociaciones, alianzas y redes implementadas; pero el éxito de estas es dependiente de cómo se articulan sus agentes, el rol que cumple cada uno de ellos y la fuerza del lazo creado; son instrumentos que ganan fuerza con el tiempo en el desarrollo local, por ejemplo, con el objetivo orientador hacia la innovación (Iturrioz, Aragón, \& Narvaiza, 2015), un cúmulo de socios permite profundizar en el aprendizaje organizacional, al conocer las diversas experiencias, y dependiendo de la capacidad de absorción, estas pueden ser empleadas para mejorar el desempeño (Zahoor \& Al-Tabbaa, 2020). A su vez, de ser posible, las propuestas deben ser analizadas en conjunto entre las partes interesadas, en la tabla 1 se extraen algunas posturas de empresarios entrevistados con relación al papel que funge el gobierno nacional, el gobierno local, de importante rol, ya que de acuerdo con (Wright, 2020) guarda un papel protagónico en su relación con las empresas locales, en especial las Pymes, debiendo mitigar el impacto del nuevo coronavirus en el empleo y la economía, que permita crear un plan de recuperación posterior al evento pandémico; de igual manera, el papel de los gremios y asociaciones ligadas al segmento en cuestión y a los mismos empresarios y sus familias, en materia de soluciones esperadas durante el periodo de cuarentena.

Tabla 1. Perspectiva del empresario con los otros sectores involucrados

\begin{tabular}{|l|l|}
\hline \multicolumn{1}{|c|}{ Sector } & \multicolumn{1}{c|}{ Descripción } \\
\hline Gobierno nacional & $\begin{array}{l}\text { "extender los alivios financieros por más tiempo", "reactivación del comercio", "apoyo } \\
\text { económico referente a los subsidios y cumpliendo con las propuestas ofrecidas", } \\
\text { "ayuda proyectada hacia los empleados", "ayudas para el pago de la nómina", "inversión, } \\
\text { estrategias de apoyo a ventas, al comercio y reducción de importaciones", "reducir el } \\
\text { número de intermediarios", "fomentar la compra nacional y reducir la compra de calzado } \\
\text { chino" o "no espero nada, prefiero hacerlo solo". }\end{array}$ \\
\hline \multirow{5}{*}{$\begin{array}{l}\text { Gobierno local } \\
\text { (alcaldías) }\end{array}$} & $\begin{array}{l}\text { "se necesita la reapertura de almacenes, es importante reactivar la economía del } \\
\text { sector", "capacitaciones a determinados sectores", "se necesita un enfoque sostenible", } \\
\text { "subsidios y eventos", "se han recibido ayudas de la Alcaldía de Cali" y "comprar } \\
\text { colombiano, nada importado" }\end{array}$ \\
\hline Gremios y asociaciones & $\begin{array}{l}\text { "charlas, conferencias y talleres", "se necesita mayor unión, ponerse la mano en el } \\
\text { corazón porque el desempleo está muy alto", "buscar soluciones, alternativas, abrir las } \\
\text { ventas y fabricar otros productos", "respaldo y apoyo para surgir como gremio", "tener } \\
\text { mucha paciencia para poder continuar" y "trabajar en conjunto" }\end{array}$ \\
\hline \multirow{5}{*}{$\begin{array}{l}\text { Empresarios y sus } \\
\text { familias }\end{array}$} & $\begin{array}{l}\text { "recortar los gastos como sea posible", "aguantar y tener mucha paciencia", "tener } \\
\text { capacidad de adaptarse", "minimizar costos", "reinventarse" o "es una situación muy } \\
\text { difícil, pero es importante ayudarle a nuestros trabajadores". }\end{array}$ \\
\hline
\end{tabular}

Fuente: elaboración propia 
Otro aspecto que reviste importancia es el uso y la implementación de la tecnología. Los empresarios se encuentran en su gran mayoría (85\%) muy interesados en la creación de una tienda virtual multimarca y en abrir un nuevo canal apoyados en el marketing digital; si se hace un acopio del pensamiento expresado, es la solución a considerar más importante en el momento y en el futuro, cuando se habla de esa nueva normalidad; algunos de los encuestados consideran viable la idea porque puede aumentar el rango de distribución a nivel nacional, se abren más puertas y se conocen más clientes; algunos de los empresarios se han logrado mantener en la actual coyuntura gracias a las redes, les ha funcionado, pero también es cierto que necesitan incursionar más en el tema. Es necesaria una profundización mediante la capacitación; tendrán una herramienta que les permita seguir compitiendo; contrariamente, otros consideran que es complicado porque hay desconocimiento, que esa tecnología es nueva para muchos, que reviste un riesgo la compra por internet, por lo que no contemplan hacerlo.

El manejo de redes y nuevas tecnologías permite acceso a nuevos mercados, reduce costos de transacción, y mejora en el rendimiento e intercambio de recursos varios, tales como bienes, dinero, información, reputación, stock, entre otros (Lin \& Lin, 2016). Existe por un lado, la necesidad de comunicación entre las MiPymes, que puedan planear un horizonte al trabajar en red, lo cual termine por fortalecer relaciones (particularmente locales) que logren engendrar una amplia gama de productos direccionados a mayoristas o minoristas (Gilmore, Carson, \& Rocks, 2006); empero, surgen problemas que parten de la comunicación entre las empresas, una articulación fallida entre socios, incertidumbre en costos asociados y pugnas por rangos de contribución, más aún cuando hay conocimiento tecnológico de por medio o los denominados "comportamientos oportunistas" (Sawers, Pretorius, \& Oerlemans, 2008).

Acá se puede hacer la salvedad en que el uso de redes sociales para promocionar productos, puede ser un mecanismo que contribuya en promover sus marcas de manera más eficiente al establecer nuevos lazos, integrando comunidades en línea y mejorando así sus oportunidades de innovación (loanid, Deselnicu, \& Militaru, 2018), de hecho, y dada la actual disrupción (tipificada como disrupción hostil por ser inesperada y exógena), los negocios se han visto abocados, de manera directa o indirecta, hacia digitalizar y transformar sus procesos y operaciones como un mecanismo adaptativo (Kamal, 2020). También es importante mantener un flujo de caja hábil y eficiente, porque la coyuntura provoca retrasos en la facturación y pago a empresas; generar la 
modificación pertinente al presupuesto y ajustarlo a las condiciones actuales; realizar acciones de conciliación automática en bancos que permita dinamizar el proceso; y, de igual forma, en relación con manejo de inventarios (Winarsih \& Fuad, 2021).

Los impactos previamente mencionados han afectado considerablemente al marketing, en el caso del orientado a la venta de productos o servicios entre empresas "business to business" o B2B, fruto del incremento del trabajo remoto, las ausencias en disponibilidad física, la cancelación de eventos como conferencias, ferias comerciales y reuniones con clientes; el cierre de fronteras entre países y las restricciones en los viajes han generado aplazamientos de las actividades esenciales de las organizaciones que terminan por complicar sus operaciones diarias (Hartmann \& Lussier, 2020). Muy palpado en la actual investigación que se lleva a cabo con el sector de calzado; hasta el momento se han logrado desarrollar algunos eventos como ferias institucionales que resaltan el carácter asociativo, por ejemplo, en UNIMINUTO (institución académica), FONPASAR (fondo de empleados), CORFEINCO (asociación mutual), Showroom con ACOPI (gremio), entre otros (ver figura 1), ejercicios que buscan incentivar mecanismos como la asociatividad, trabajo colaborativo y búsqueda de otros canales de comercialización, los cuáles han tenido que ser aplazados por la contingencia presentada, abriendo el panorama para modificar la gestión y acompañamiento hacia el entorno virtual. Actualmente, y dado que la coyuntura obedece a un episodio impredecible, la toma de decisiones ha sido compleja, derivando en un cambio sustancial, donde la carencia en la información y comprensión de lo observado termina por proyectar incapacidad de predecir el impacto de las acciones que las empresas tomen (Maya \& Jie, 2020). 

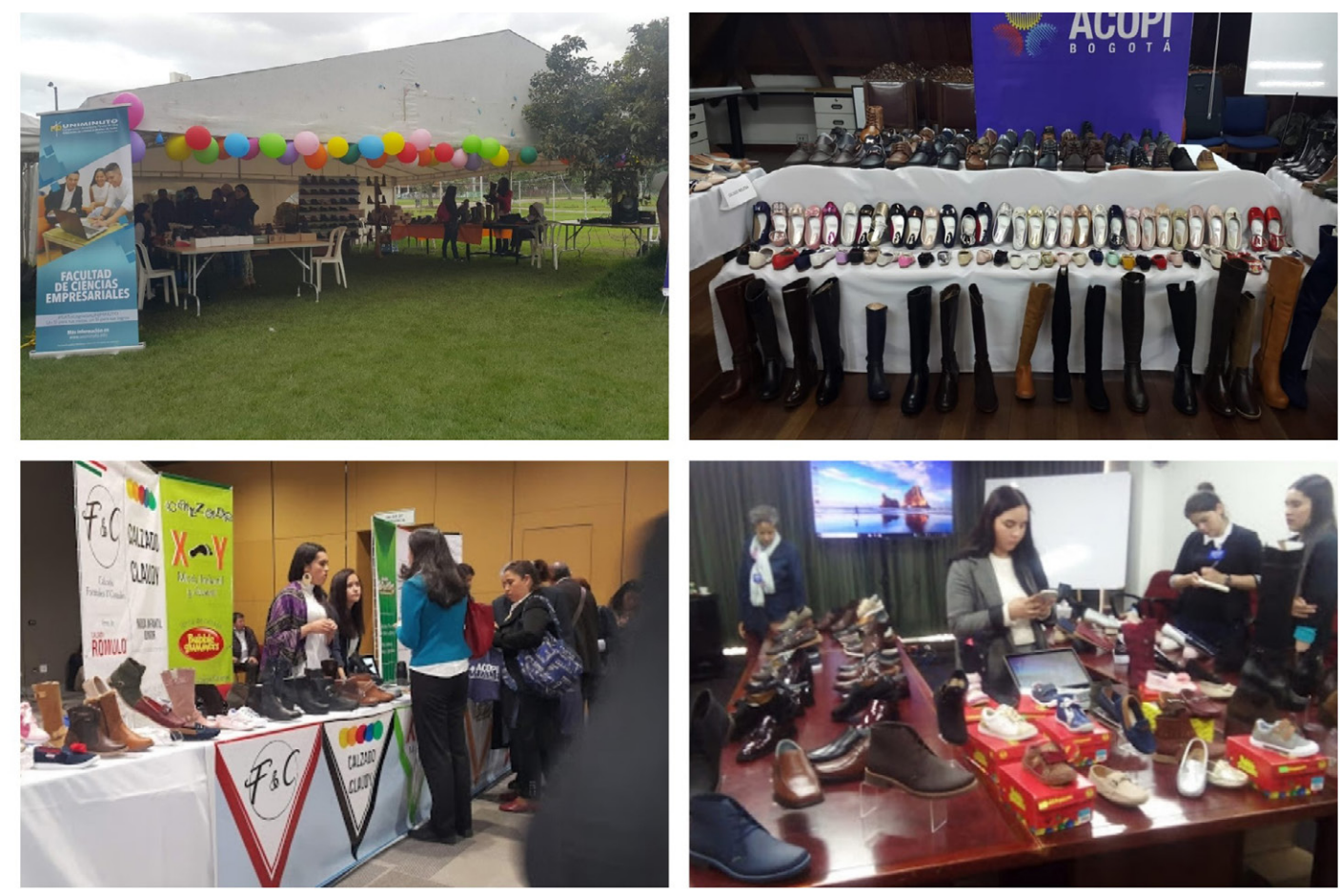

Figura 1. Algunos eventos propuestos con los empresarios del calzado previa aparición de la pandemia.

Fuente: autores (2018 - 2019)

Finalmente, se considera pertinente establecer el empalme de los planteamientos propuestos por (Rapaccini, Saccani, Kowalkowski, Paiola, \& Adrodegari, 2020), en donde se esgrime un modelo que permite apoyar la gestión ante la disruptiva que trae consigo el fenómeno pandémico. En su primera fase "Calamity", se inicia comprendiendo el fenómeno, por lo que se propone activación de grupos de trabajo para el manejo de la crisis, generar consciencia, elaborar escenarios y evaluar sus recursos, tecnologías y conocimiento que pueda decantar en prácticas; una segunda fase "Quick \& Dirty" propende por adoptar protocolos de salud y seguridad en los trabajadores, reduce operaciones y colabora con socios y clientes para encontrar soluciones que superen las restricciones; una tercera fase "Restart" en concordancia con protocolos, por ejemplo, de manejo de protección personal, distancia social, entrenamiento y rastreo, que esté lista para el cumplimiento en incrementos de demanda por parte del cliente, o que le permita recuperarse en casos de nuevas limitaciones o turbulencias en el mercado; y finalmente, una cuarta fase "Adapt to Next Normal" comprendiendo los nuevos cambios que afecten entornos económicos, sociales y culturales, ubicando nuevos caminos. 


\section{CONCLUSIONES}

Con el arribo del suceso pandémico, las actividades económicas se han visto perjudicadas, y dentro de la taxonomía empresarial, las MiPymes son las que han recibido el impacto más severo. En el actual escrito se hace una aproximación al sector del calzado con relación al impacto recibido por las medidas de distanciamiento social adoptadas desde el gobierno; se identifica la clara repercusión en sus operaciones frente a un evento externo no esperado, su capacidad de maniobra se ve afectada por lo que resulta poco sostenible en el marco temporal mantener el nivel de producción y ventas, recortando así el flujo de efectivo; lo que deriva en una ralentización de los procesos, reducción del volumen de mano de obra y cierres temporales o definitivos.

El estudio presentó ciertas limitaciones en la recolección de la información, algo que puede considerarse normal dada la alteración observada, no obstante, resulta imperativo que se hilvane el ejercicio académico y gubernamental con la puesta en escena de prácticas (como, por ejemplo, las tecnológicas) que mejore las expectativas de las MiPymes de este sector, un entorno económico con gran incertidumbre.

\section{REFERENCIAS}

ACICAM. (2020). ¿Cómo va el sector? Enero a diciembre 2019. Bogotá. https://acicam.org/download/ como-va-el-sector-diciembre-2019-2/

ACOPI. (2020). Tablero de control impacto del Covid-19 en las MiPyme de Bogotá. Bogotá D. C. http:// observatorio.desarrolloeconomico.gov.co/emprendimiento/tablero-de-control-impacto-covid19-mipyme-bogota-junio-21

Bairagi, V., \& Munot, M. V. (2019). Research Methodology, a practical and Scientific Approach. New York: CRC Press.

Bourletidis, K., \& Triantafyllopoulos, Y. (2014). SMEs Survival in time of Crisis: Strategies, Tactics and Commercial Success Stories. Procedia - Social and Behavioral Sciences, 148, 639-644. https://doi. org/10.1016/j.sbspro.2014.07.092 
Brown, R., \& Rocha, A. (2020). Entrepreneurial uncertainty during the Covid-19 crisis: Mapping the temporal dynamics of entrepreneurial finance. Journal of Business Venturing Insights, 14. https:// doi.org/10.1016/j.jbvi.2020.e00174

Cámara de Comercio de Bogotá. (junio de 2016). Cuero, Calzado y Marroquinería sector de talla mundial. https://www.ccb.org.co/Clusters/Cluster-de-Cuero-Calzado-y-Marroquineria/Noticias/2016/ Junio-2016/Cuero-Calzado-y-Marroquineria-sector-de-talla-mundial

Cámara de Comercio de Bogotá. (Octubre de 2019). Industria del cuero apuesta por cuidado del medio ambiente. https://www.ccb.org.co/Clusters/Cluster-de-Cuero-Calzado-y-Marroquineria/ Noticias/2019/Octubre-2019/Industria-del-cuero-apuesta-por-cuidado-del-medio-ambiente

Chakraborty, I., \& Maity, P. (2020). COVID-19 outbreak: Migration, effects on society, global environment and prevention. Science of the Total Environment, 728. https://doi.org/10.1016/j. scitotenv.2020.138882

Confente, I., Buratti, A., \& Russo, I. (2015). The role of servitization for small firms: drivers versus barriers. International Journal of Entrepreneurship and Small Business, 26(3), 312-331. 10.1504/ IJESB.2015.072394

Eggers, F. (2020). Masters of disasters? Challenges and opportunities for SMEs in times of crisis. Journal of Business Research, 116, 199-208. https://doi.org/10.1016/j.jbusres.2020.05.025

Elavarasan, R. M.,\& Pugazhgendhi, R. (2020). Restructured society and environment: A review on potential technological strategies to control the COVID-19 pandemic. Science of the Total Environment, 725. https://doi.org/10.1016/j.scitotenv.2020.138858

Gilmore, A., Carson, D., \& Rocks, S. (2006). Networking in SMEs: Evaluating its contribution to marketing activity. International Business Review, 15(3), 278-293. https://doi.org/10.1016/j. ibusrev.2006.02.003 
Hartmann, N. N., \& Lussier, B. (2020). Managing the sales force through the unexpected exogenous COVID-19 crisis. Industrial Marketing Management, 88, 101-111. https://doi.org/10.1016/j. indmarman.2020.05.005

Ioanid, A., Deselnicu, D. C., \& Militaru, G. (2018). The impact of social networks on SME's innovation potential. Procedia Manufacturing, 22, 936-941. https://doi.org/10.1016/j.promfg.2018.03.133

Iturrioz, C., Aragón, C., \& Narvaiza, L. (2015). How to foster shared innovation within SMEs' networks: Social capital and the role of intermediaries. European Management Journal, 33(2), 104-115. https:// doi.org/10.1016/j.emj.2014.09.003

Kamal, M. M. (2020). The triple-edged sword of COVID-19: understanding the use of digital technologies and the impact of productive, disruptive, and destructive nature of the pandemic. Information Systems Management. https://doi.org/10.1080/10580530.2020.1820634

Kottika, E., Özsomer, A., Rydén, P., Theodorakis, I. G., Kaminakis, K., Kottikas, K. G., \& Stathakopoulos, V. (2020). We survived this! What managers could learn from SMEs who successfully navigated the Greek economic crisis. Industrial Marketing Management, 88, 352-365. https://doi.org/10.1016/j. indmarman.2020.05.021

Lin, F. J., \& Lin, Y. H. (2016). The effect of network relationship on the performance of SMEs. Journal of Business Research, 69(5), 1780-1784. doi:https://doi.org/10.1016/j.jbusres.2015.10.055

Maya, B., \& Jie, F. (2020). Analysis of the uncertainty sources and SMEs' performance. Journal of Small Business \& Entrepreneurship. https://doi.org/10.1080/08276331.2020.1764737

Mol-Gómez-Vázquez, A., Hernández-Cánovas, G., \& Köeter-Kant, J. (2020). Economic and institutional determinants of lease financing for European SMEs: An analysis across developing and developed countries. Journal of Small Business Management. https://doi.org/10.1080/00472778.2020.1800352 
OMS. (16 de Marzo de 2020). Declaración conjunta de la ICC y la OMS: Un llamamiento a la acción sin precedentes dirigido al sector privado para hacer frente a la COVID-19. https://www.who.int/es/ news-room/detail/16-03-2020-icc-who-joint-statement-an-unprecedented-private-sector-callto-action-to-tackle-covid-19

Páez, P. N., Jiménez, W. G., \& Danna-Buitrago, J. P. (2018). La competitividad de los artículos de calzado, cuero y marroquinería en Colombia: revisión de la literatura. Revista Diálogos de Saberes(48), 171196. https://doi.org/10.18041/0124-0021/dialogos.48.2018.4729

Papadopoulos, T., Baltas, K. N., \& Balta, M. E. (2020). The use of digital technologies by small and medium enterprises during COVID-19: Implications for theory and practice. International Journal of Information Management. https://doi.org/10.1016/j.ijinfomgt.2020.102192

Rapaccini, M., Saccani, N., Kowalkowski, C., Paiola, M., \& Adrodegari, F. (2020). Navigating disruptive crises through service-led growth: The impact of COVID-19 on Italian manufacturing firms. Industrial Marketing Management, 88, 225-237. https://doi.org/10.1016/j.indmarman.2020.05.017

Rivera, J. A. (2020). Rendimiento contable y EVA en la pyme de la industria del cuero, calzado y marroquinería en Colombia. Universidad \& Empresa, 22(38), 131-159. https://doi.org/10.12804/ revistas.urosario.edu.co/empresa/a.7335

Sawers, J. L., Pretorius, M. W., \& Oerlemans, L. A. (2008). Safeguarding SMEs dynamic capabilities in technology innovative SME-large company partnerships in South Africa. Technovation, 28(4), 171182. https://doi.org/10.1016/j.technovation.2007.09.002

Shah, A. U., Azrie, S., Thevadas, R., Noordin, N. K., Rahman, A., Sekawi, Z., . . Hameed, M. T. (2020). COVID-19 outbreak in Malaysia: Actions taken by the Malaysian government. International Journal of Infectious Diseases, 97, 108-116. https://doi.org/10.1016/j.ijid.2020.05.093 
Winarsih, I. M., \& Fuad, K. (2021). Impact of Covid-19 on Digital Transformation and Sustainability in Small and Medium Enterprises (SMEs): A Conceptual Framework. En L. Barolli, A. PoniszewskaMaranda, \& T. Enokido, Complex, Intelligent and Software Intensive Systems. CISIS 2020. Advances in Intelligent Systems and Computing. Springer, Cham. https://doi.org/10.1007/978-3-030-504540_48

Wright, C. (2020). Local government fighting Covid-19. The Round Table, 109(3), 338-339. https://doi.or $\mathrm{g} / 10.1080 / 00358533.2020 .1763017$

Zahoor, N., \& Al-Tabbaa, O. (2020). Inter-organizational collaboration and SMEs' innovation: A systematic review and future research directions. Scandinavian Journal of Management, 36(2). https://doi. org/10.1016/j.scaman.2020.101109 